\title{
RESEARCH ARTICLE \\ On principal eigenpair of temporal-joined adjacency matrix for spreading phenomenon
}

\author{
Shih-Chieh Wang ${ }^{1}$ (D) Nobuyasu Ito ${ }^{1,2}$
}

Received: 26 December 2018 / Accepted: 8 January 2019 / Published online: 28 January 2019

(c) The Author(s) 2019

\begin{abstract}
This paper reports a framework of analysis of spreading herbivore of individualbased system with time evolution network $\widetilde{A}(t)$. By employing a sign function $\theta_{1}(x)$, $\theta_{1}(0)=0, \theta_{1}(x)=1 x \in \mathbb{N}$, the dynamic equation of spreading is in a matrix multiplication expression. Based on that, a method of combining temporal network is reported. The risk of been-spread and the ability to spread can be illustrated by the principal eigenpair of temporal-joined matrix in a system. The principal eigenpair of post-joined matrix can estimate the step number to the farthest agent $S_{i}$ in a non-time evolution network system $\widetilde{A}(t)=\widetilde{A}$ as well.
\end{abstract}

Keywords Agent model $\cdot$ Epidemic $\cdot$ Contact network

\section{Introduction}

Various applications of network are applied crossing social and natural disciplinaries [1]. As a mathematical abstraction method, network describes the interactions among elements inside of system as links among nodes. The non-direction and static network gets the highest abstraction level and gives us uncountable results of explaining the dynamic properties of system. Temporal network [2] reduced the level of abstraction to contain essential dynamic information of system. Spectral method and eigensystem decomposition of network adjacency matrix, or of Laplacian matrix, are for the word abstraction method, which reveals the topology properties of network [3] and dynamic properties of system [3, 4]. But we do not have a good framework to combine spectral method and temporal network nowadays. Spreading on network is a scientific problem that wants such framework most. A

Shih-Chieh Wang

shih-chieh.wang@riken.jp

1 RIKEN Center for Computational Science, 7 Chome-1-26 Minatojima Minamimachi, Chuo Ward, Kobe, Hyōgo Prefecture 650-0047, Japan

2 Department of Applied Physics, School of Engineering, The University of Tokyo, 7-3-1 Hongo, Bunkyo-ku, Tokyo 113-8656, Japan 
query on the Thompson Web of Science database, more 1500 papers for the year 2017, shows the importance of spreading in complex network. But the situation of eigensystem explanation for spreading problem does not go well. Valdano et al. reviewed past works and left a negative comment for applying eigenvector centralities method from static contacting network in year 2015 [5], they used statistics of contacting data.

This paper is organized as follows: the next section shows the dynamic equation of spreading. Following that the numerical result of non-time evolution network is shown; followed by the spreading speed of periodical repeating temporal network. Future work is followed by the discussion section. Derivation of the dynamic equation is arranged in the "Appendix".

\section{Matrix representation formula for spreading via network}

For an individual-based simulation, one single-network site indicates one agent only. We use binary value $(\vec{H}(t))_{i}$ for describing state of been spread for $i$ th agent at time $t$. While the value of $(\vec{H}(t))_{i}$ is zero it indicates the situation that the $i$ th agent is in freeform been-spread state, and value one for been-spread state, respectively. The only condition of turning to be been-spread state of each agent is the existence of beenspread neighbour. Since an agent is in been-spread state, this been-spread agent will remain in this state forever. We formulate this spreading dynamic of system with $N$ agents on time as an evoluting network:

$$
\vec{H}(t)=\theta_{1}\left(\left(\prod_{t^{\prime}=0}^{\curvearrowleft}\left(\widetilde{I}+\widetilde{A}\left(t^{\prime}\right)\right) \vec{H}(0)\right),\right.
$$

$\widetilde{A}(t)$ is an adjacency matrix representation for temporal network at time $t, \widetilde{A}(t)_{i, j}=1$ means that $i$ th and $j$ th agent is connected with a unidirectional link at time $t$, the value zero $\widetilde{A}(t)_{i, j}=0$ means not linked, respectively. $\widetilde{I}$ is a $N \times N$ identity matrix. The symbol $\prod^{\curvearrowleft}$ is left-matrix-product notation, $\prod_{l=1}^{n} \widetilde{A_{l}}=\widetilde{A_{m}} \cdots \widetilde{A_{2}} \widetilde{A_{1}}$. Where function $\theta_{1}(x)$ is a simplified form denoting unit step function, $\theta_{1}(x+1)=\theta(x)$. The "Appendix" shows the properties of $\theta_{1}(x)$. The derivation of Eq. (1) is shown in the "Appendix" with these $\theta_{1}(x)$ s properties.

\section{Non-time-evolution matrix}

The first step to reveal the meaning of eigensystem is choosing the most simple casenon-evolution network and single spreading source, $\widetilde{A}(t)=\widetilde{A},(\vec{H}(0))_{j}=\delta_{i, j}$. The $i$ th agent is the unique spreading source. In the non-evolution network case, the Eq. (1) can be simplified as $\vec{H}(t)=\theta_{1}\left((\widetilde{I}+\widetilde{A})^{t} \vec{H}(0)\right)$, by employing Eq. (13). The been-spread 
state of $j$ th agent at time $t$ will be $\theta_{1}\left(\left((\widetilde{I}+\widetilde{A})^{t}\right)_{i j}\right)$. This condition is the $100 \%$ beenspread starting from $i$ th agent as a unique spreading origin:

$$
\left((\widetilde{I}+\widetilde{A})^{t}\right)_{i j} \geq 1, \forall j .
$$

Eigenmode decomposition is more easy to comprehend for this matrix multiplication, $\left.\left.(\widetilde{I}+\widetilde{A})^{t}\right)_{i j}=\sum_{k}\left(\left(\lambda_{k}+1\right)^{t} w_{i, k} w_{j, k}\right)\right)$. The $k$ th eigenpair of matrix $\widetilde{A}$ is $\lambda_{k}$ and $\vec{W}_{k}$ is, such eigenpairs satisfy the equation $\widetilde{A W}_{k}=\lambda_{k} \vec{W}_{k}$. The $i$ th agent's eigenvector component in $k$-eigenvector is $w_{i, k}, w_{i, k}=\left(\vec{W}_{1}\right)_{k}$ The eigenpair indexes $k$ are arranged as a descending order: $\lambda_{1} \geq \lambda_{2} \geq \cdots \geq \lambda_{N}$. While $\underset{\sim}{k}=\underset{\sim}{\sim}, \lambda_{\mathcal{L}}$ and $\underset{\sim}{\sim} \underset{i, 1}{ }$ are called the principal eigenpair. The three similar matrices, $(\widetilde{I}+\widetilde{A})^{t}, \widetilde{I}+\widetilde{A}$ and $\widetilde{A}$ share the same eigenvector set. While condition:

$$
\left(\left(\lambda_{1}+1\right) /\left(\lambda_{2}+1\right)\right)^{t} \gg 1
$$

the principal eigenpair is suitable for estimating lower bound of $S_{i}$ :

$$
E^{\text {lower }}\left(S_{i}\right)=-\frac{\log \left(w_{i, 1} w_{j^{\min }, 1}\right)}{\log \left(\lambda_{1}+1\right)} \text {. }
$$

\section{Case 1: Spanning tree}

If this non-time evolution network is spanning tree, the first agent, $i=1$, is the hub of this spanning tree, other agents link to the hub and there was no other link in this network. The off-diagonal elements of adjacency matrix are $\widetilde{A}_{i j}=\widetilde{A}_{i j}=1$ if $i=1$. In this network, $S_{1}=1$ for $i=1$, and $S_{i}=2$ for others. For understanding the asymptotic behaviour when system size goes to large $N \rightarrow \infty$, we denote a symbol $1 / \alpha^{2}=N-1$. The process of getting principal eigenpair of $\widetilde{A}, \lambda_{1}$ and $\vec{W}_{1}$, is following: to solve the eigenvector equation $\widetilde{A}_{1}=\lambda_{1} \vec{W}_{1}$ with (a) positive eigenvector assumption $w_{i, 1}=\left(\vec{W}_{1}\right)_{i}>0$; (b) network symmetric $w_{j, 1}=w_{l, 1} \forall j, l>2$; (c) eigenvector normalization $\sum_{i} w_{i, 1}^{2}=1$. The principal eigenpair is $\lambda_{1}=1 / \alpha$,

$$
w_{i, 1}=\left\{\begin{array}{ll}
\frac{1}{\sqrt{\alpha^{4}+1}} & \text { if } i=1 \\
\frac{\alpha}{\sqrt{\alpha^{4}+1}} \text { else }
\end{array} .\right.
$$

We can calculate the $E^{\text {lower }}\left(S_{i}\right)$ of each agent from Eq. (4):

$$
E^{\text {lower }}\left(S_{i}\right)=\left\{\begin{array}{ll}
-\frac{\log \left(\frac{\alpha}{\alpha^{4}+1}\right)}{\log \left(\frac{1}{\alpha}+1\right)} \approx 1+\frac{\alpha}{\log (\alpha)}+O\left(\alpha^{2}\right) \quad \text { if } i=1 \\
-\frac{2 \log \left(\frac{\alpha}{\sqrt{\alpha^{4}+1}}\right)}{\log \left(\frac{1}{\alpha}+1\right)} \approx 2+\frac{2 \alpha}{\log (\alpha)}+O\left(\alpha^{2}\right) \text { else }
\end{array} .\right.
$$


Such Taylor expansion shows the asymptotic behaviour when $N \rightarrow \infty$ as $\alpha \rightarrow 0$. The Fig. 1 shows the numerical result of it.

\section{Case 2: Circular loop and it with and without an radius link}

The estimation formula Eq. (4) contains symmetric properties from the original network. We choose two networks to compare to state that-circular loop and it with and without a radius link. In the network with a radius link, a topological symmetric of rotation will be break. It is a $N=16$ one dimensional loop, the degree (neighbours) of each agent is two. Such loop network contains symmetric properties and also can be found in the principal eigenvector components: $w_{i, 1}=1 / \sqrt{N}$. The step number to coverage all the network should be half size of network, $S_{i}=N / 2=8$. This result can be visualized as non zero matrix element figure of matrix $(\widetilde{I}+\widetilde{A})^{t}$ in Fig. 2 . The value of estimation of $S_{i}$ of each agent from principal eigenpair in Eq. (4) is the same:

$$
E^{\text {lower }}\left(S_{i}\right)=\frac{\log N}{\log 3} \approx 2.52372,
$$

where the principal eigenvalue is equal to average degree of network, $\lambda_{1}=2$. There is no asymptotic behaviour when system size goes to infinity here, $\lim _{N \rightarrow \infty} E^{\text {lower }}\left(S_{i}\right) \neq S_{i}$, because the $\lim _{N \rightarrow \infty} \lambda_{1} / \lambda_{2}=\lim _{N \rightarrow \infty} 1 /(\cos (2 \pi / N))=1$. That does not fit the condition of $E^{\text {lower }}\left(S_{i}\right.$ ) in Eq. (3). Such asymptotic eigenvalue degeneracy will be broken when we add a radius link. The radius link links first agent and $N / 2+1$ th agent. The spectral properties can be calculated as a perturbation problem when system size goes to large. Form the fist step of perturbation calculation, the value of $\lambda_{1}, w_{1,1}$ and $w_{1, N / 2+1}$ is larger then them in the simple loop, then the estimation values of $S_{i}, S_{1}$ and $S_{N / 2+1}$ become smaller. The calculation of eigensystem of this small system does not need perturbation method. The estimation value of $S_{i}$ from principal eigenpair of this system is shown in Fig. 3.

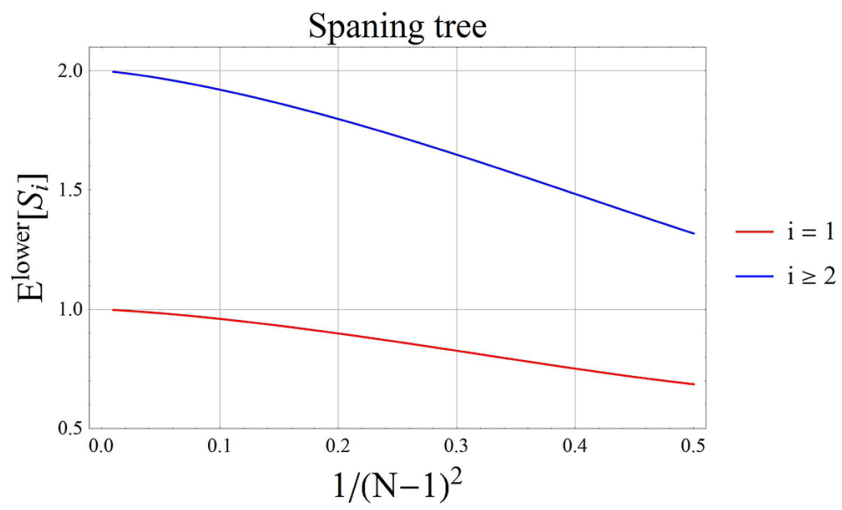

Fig. 1 Estimating step number to the end of each agent of spanning tree case. A one-level spanning tree is that hub agent, with the index $i=1$, link all other agent $i=2 \sim N$. In this network, $S_{1}=1$ for $i=1$, and $S_{i}=2$ for others. Eq. (4) is estimation formula that was used. This numerical result shows an asymptotic behaviour when system size goes to large. The analytical form is shown in Eq. (6) 


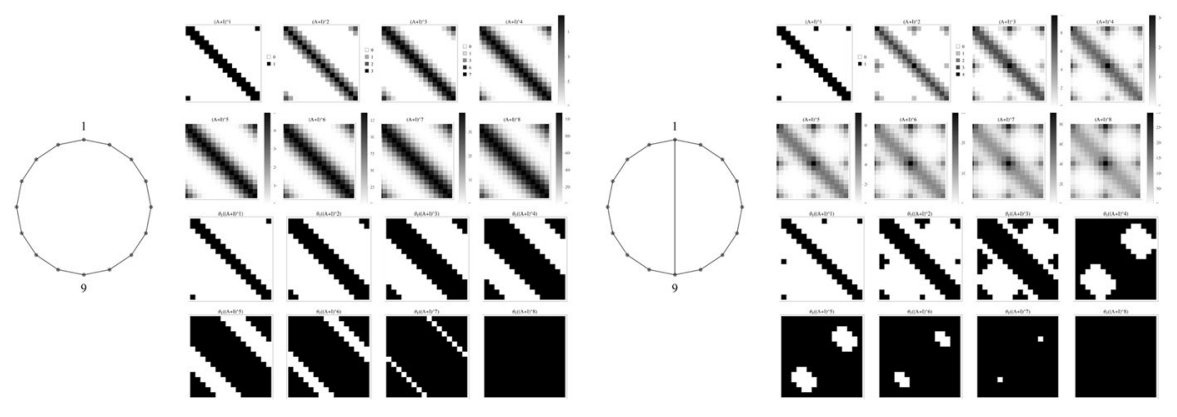

Fig. 2 Matrix multiplication of adjacency matrix. Using this multiplication matrix post mapped by the function $\theta_{1}(x)$, shown as black and white, the step number to the furthest agent can be obtained. In the left panel as a network without radius link, all columns or rows turn to black $\theta_{1}\left((\widetilde{A}+\widetilde{I})^{t}\right)_{i j}=1$ while $t=8$. Before that, at least one element in row is white. White matrix element $\theta_{1}\left((\widetilde{A}+\widetilde{I})^{t}\right)_{i j}=0$ means that $j$ th agent can not be accessed by $i$ th agent. Therefore, $S_{i}=8$ for all agents in the loop network without radius link. There are two white matrix elements in the loop network with a radius link at $t=7$. The two matrix elements are $\theta_{1}\left((\widetilde{A}+\widetilde{I})^{7}\right)_{5,13}=\theta_{1}\left((\widetilde{A}+\widetilde{I})^{7}\right)_{13,5}=0$. It makes that $S_{i}=8$ for $i=13$ and 5 . Other agents' $S_{i}$ is shown in "Non-evolution matrix". Premapped matrix by the function $\theta_{1}(x)$, shown as grey level, does not show $S_{i}$ information clearly

Fig. 3 Estimation value of $S_{i}$ for the loop network with a radius link. The agent eigenvector component homogeneity in principal eigenpair among simple loop system is broken down by adding a radius link. But some systematic properties that $S_{i}$ has remains. That is the reason that why the estimation value of $S_{i}$ in the system of $N=16$ in this figure forms five points only. The value of $S_{i}$ comes from Fig. 2. The estimation value of $S_{i}$ comes from Eq. (4). We discuss this figure in "Non-evolution matrix"

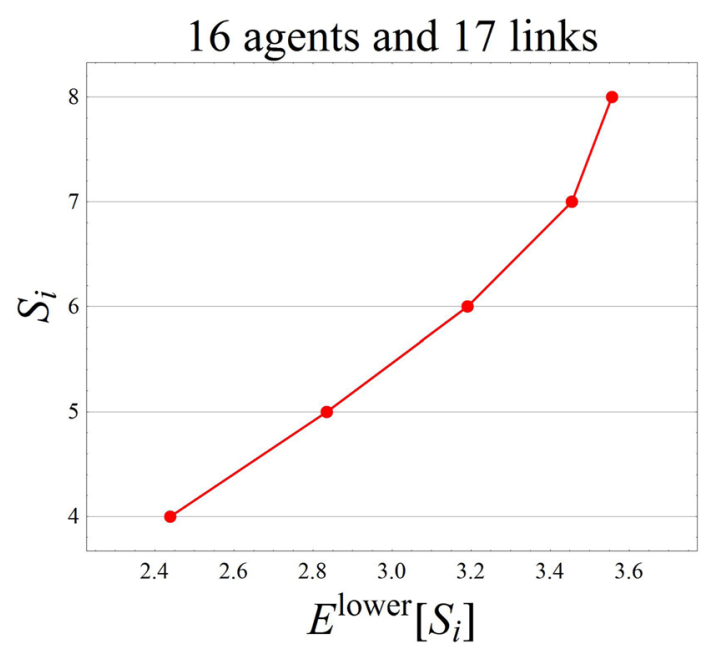

The $S_{i}$ have three kind of symmetric properties, our estimation also shows the same symmetric properties:

$$
\begin{gathered}
S_{1}=S_{n / 2+1}, \\
\left\{\begin{array}{ll}
S_{1+l}=S_{n-1-l} \quad l=1,2, \ldots, N / 2 \\
S_{n / 2+1+l}=S_{n / 2+1+n-1} & l=0,1 \ldots, N / 2
\end{array} .\right.
\end{gathered}
$$

Combining the three symmetric properties, the system of $N$ will partition as four groups, within the edge node of group, the $S_{i}$ s have $N / 4+1$ values. Therefore, the agents in the same set share the same value of $S_{i}:\{1,9\},\{2,16,10,8\},\{3,15,11,7\}$, 
$\{4,14,12,6\},\{5,13\}$. The results of Fig. 2 state that: $S_{i}=3+i \quad i=1-5$, we can understand them from the following easy examples. In this network, the distance to the farthest agent of 5th and of 13th agent is the same as them in the loop network without radius link $A_{1, N / 2+l}$. The value of $S_{i}$ for 5 th and of 13 th agent remains the same $S_{5}=S_{13}=8$. For the 4th agent, the distance to the farthest agent, 12th, gets one step smaller by shifting to the route with the radius link, from the route $\{4 \rightarrow 3 \rightarrow 2 \rightarrow 1 \rightarrow 16 \rightarrow 15 \rightarrow 14 \rightarrow 13 \rightarrow 12\}$ to the route $\{4 \rightarrow 3 \rightarrow 2 \rightarrow 1 \rightarrow 9 \rightarrow 10 \rightarrow 11 \rightarrow 12\}$. These symmetric properties can also be found in our estimator because they are in the principal eigenvector, that can be revealed by calculating higher order perturbations. These correspondences of symmetry are shown in Fig. 3 as five $\{x, y\}$ points, otherwise it will show more than five points. Comparing to the relation of $S_{i}$ and its lower bound estimator $E^{\text {lower }}\left(S_{i}\right)$, our estimator has the network symmetric properties and the monotonic relation to $S_{i}$.

\section{Temporal evolution network}

For a system that the networks within $\widetilde{A}(t)$ repeat each $\tau$ step, $\widetilde{A}(t)=\widetilde{A}(t+\tau)$, the time evolution of been-spread state $\vec{H}(t)$ in Eq. (1) can be denoted by a $\widetilde{P}$ matrix: $\vec{H}(t)=\theta_{1}\left((\widetilde{P})^{t / \tau} \vec{H}(0)\right)$, where

$$
\widetilde{P}=\theta_{1}\left(\left(\prod_{t^{\prime}=0}^{\curvearrowleft}\left(\widetilde{I}+\widetilde{A}\left(t^{\prime}\right)\right)\right) .\right.
$$

The following will show the $\widetilde{P}$ and its principal eigenpair for two artificial cases, $N=3$ and $N=60$, respectively. The results show that principal eigenpair of $\widetilde{P}$ carries the dynamic information during the period $\tau$.

\section{$N=3, \tau=2$ case}

Each time step contains one link of the route from first agent to the third agent via the second agent. The first step links the first and the second agent: $(\widetilde{A}(0))_{1,2}=(\widetilde{A}(0))_{2,1}=1$, and $(\widetilde{A}(0))_{i, j}=0$ for else $\{i, j\}$ pair. The second step links the second and the third agent: $(\widetilde{A}(1))_{2,3}=(\widetilde{A}(1))_{3,2}=1$, and $(\widetilde{A}(1))_{i, j}=0$ for else $\{i, j\}$ pair. The matrix expression of network $\widetilde{A}\left(t^{\prime}\right)$ is shown in the process of getting matrix $\widetilde{P}$ :

$$
\begin{aligned}
\widetilde{P}=\theta_{1}((\widetilde{A}(1)+\widetilde{I}) & (\widetilde{A}(0)+\widetilde{I}))=\theta_{1}\left(\left(\begin{array}{lll}
1 & 0 & 0 \\
0 & 1 & 1 \\
0 & 1 & 1
\end{array}\right)\left(\begin{array}{lll}
1 & 1 & 0 \\
1 & 1 & 0 \\
0 & 0 & 1
\end{array}\right)\right) \\
\rightarrow \widetilde{P} & =\left(\begin{array}{lll}
1 & 1 & 0 \\
1 & 1 & 1 \\
1 & 1 & 1
\end{array}\right) .
\end{aligned}
$$


The three eigenvalues of matrix $\widetilde{P}$ in descending order are $\left\{\frac{1}{2}(3+\sqrt{5}), \frac{1}{2}(3-\sqrt{5}), 0\right\}$. Their corresponding eigenvectors will be $\quad\left\{\left\{\frac{1}{2}(\sqrt{5}-1), 1,1\right\},\left\{\frac{1}{2}(-1-\sqrt{5}), 1,1\right\},\{-1,1,0\}\right\}$ and equal to $\{\{0.618034,1 ., 1\},.\{-1.61803,1 ., 1\},.\{-1 ., 1 ., 0\}\}$ numerically. Comparing with the value of $w_{1,1}$, the larger eigenvector component in principal eigenpair of second and of third agent indicates they have higher risk of been-spread. This result can also tell from the $\widetilde{A}\left(t^{\prime}\right)$ : the second agent and the third agent receives the spreading from other two agents, but the first agent cannot receive the spreading from the third agent. It is fair that there is no degree of data compression during the process to get matrix $\widetilde{P}$ from $\widetilde{A}\left(t^{\prime}\right)$, six binary number pre and post the process. It is notable that a meaning of matrix elements of matrix $\widetilde{P}$ 's transpose matrix $\widetilde{P}^{\mathrm{T}}$ is the possible agentspreading from. Larger values of $\widetilde{P}^{\mathrm{T}}$, s principal eigenvector component show more ability to spread things to other agents. That can be shown by this ration value in this system $w_{1,1} / w_{3,1}=w_{2,1} / w_{3,1}=1.61$.

\section{$N=60, \tau=5$ case}

Permutation order of network in time will not change the average value in time, but it changes the result of spreading. This part shows a very simple example that the time order of network appearing changes the spreading importance of each agent. There are only two networks in this system, $\widetilde{A^{\# 1}}$ and $\widetilde{A^{\# 2}}$, and only one network appears in each time step. The two networks and their relation can be understood as following procedure. First, loop the $N=60$ agents as a ring. Partition this ring loop into $N_{\text {group }}=10$ groups by taking $N_{\text {group }}$ inter-group links. These $N_{\text {group }}$ inter-group links form network \#2, $\widetilde{A^{\# 2}}$. The other $\left(N-N_{\text {group }}\right)$ intra-group links form network \#1, $\widetilde{A^{\# 1}}$. That

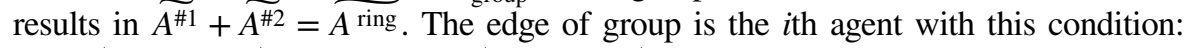
$\bmod \left(i, N / N_{\text {group }}\right)=1$, or mod $\left(i, N / N_{\text {group }}\right)=0$. For example, first, 6th and 60th agents are the edge agents. We discuss two kind of permutation orders in a $\tau=5$ window, $\widetilde{A^{\# 2}}$ first and $\widetilde{A^{\# 2}}$ last. In the first kind of permutation order, $\widetilde{A^{\# 2}}$ first, network $\widetilde{A^{\# 2}}$ $\underset{\widetilde{A} p e a r s}{ }$ in the first step $\widetilde{A}(0)=\widetilde{A^{\# 2}}$, and $\widetilde{A^{1}}$ appears in the following next four steps, $\widetilde{A}(t)=\widetilde{A^{\# 1}} t=1-4$. This permutation order repeats every $\tau=5$ steps: $\widetilde{A}(t)=\widetilde{A}(t-\tau)$. The beginning position of permutation order of network $\# 2, \widetilde{A}(0)=\widetilde{A^{\# 2}}$, helps the edge agents spread to his neighbourhood group. Other non-edge can spread to his neighbourhood group since next $\tau$ is repeating. The stronger spread ability to other agents of edge agents has been confirmed by the magnitude ratio principal eigenvector components shown in Fig. 4 as blue points. In the second permutation order, the inter-group link was placed in the last $\widetilde{A}(4)=\widetilde{A^{\# 2}}$, the ability of spreading of edge agents is suppressed. This result can also be understood from a perspective of principal eigenvector components in Fig. 4 as orange points. In summary, the principal eigenvector components of matrix $\widetilde{P}$ or $\widetilde{P}^{\mathrm{T}}$ contain the information we need, the ability of spreading and the risk of been-spread, respectively. This eigenvector representation is highly compressed. Post the normalization of eigenvector components, $\sim w_{i, 1}^{2}=1$, we use $N-1$ numbers to represent the information among $\tau N / 2$ binary numbers. 


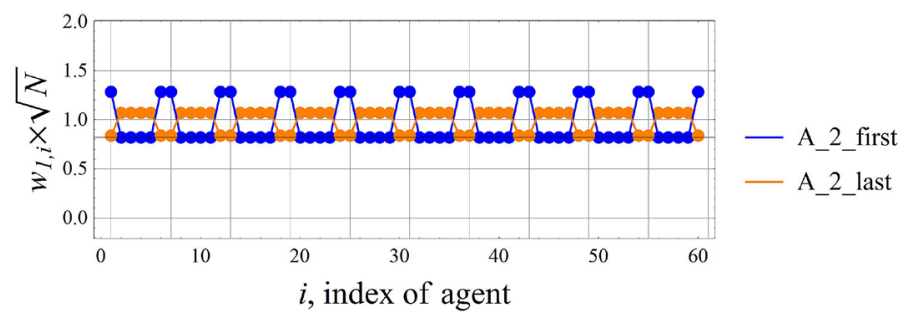

Fig. 4 Matrix $\widetilde{P}^{\mathrm{T}}$ 's principal eigenvector component. The related magnitude of edge agents' principal eigenvector components to non-edge one rises while pushing the appearance time of inter-group-edgenetwork $\widetilde{A^{\# 2}}$ in $\tau$ periodically repeat time window from the last position (orange colour) to the first place (blue colour). The first agent $i=1$ is a typical example for edge agent, and $i=2$ is non-edge one. That means that the related spreading ability increases for group-edge agents. The matrix $\widetilde{P}^{\mathrm{T}}$ is evaluating from the Eq. (10), notation ${ }^{\mathrm{T}}$ for matrix transpose

\section{Conclusion}

Including highly transitive disease, a generalized formalism of dynamic equation of spreading phenomena in a matrix multiplication expression is shown in Eq. (1). That dynamic equation also states the importance of principal eigenpair. In a non-time evolution network system $\widetilde{A}(t)=\widetilde{A}$, principal eigenpair can estimate the step number to the farthest agent $S_{i}$. In a time evolution network system $\widetilde{A}(t)$, the risk of been-spread and the ability to spread is illustrated by the principal eigenvector of matrix $\widetilde{P}$ and of its transposed one $\widetilde{P}^{\mathrm{T}}$, respectively.

We find the asymptotic degeneracy for principal eigenvalues in "Derivation of the formula". How the other eigenpair and degeneracy impact spreading phenomena is arranged in our recent studies. We also will apply this method for studying the various epidemic model besides traditional compartmental epidemic agent models [6] and for super-spreading phenomena and target vaccine problem.

Acknowledgements This research was supported by Japan MEXT as Exploratory Challenges on Post-K computer (Studies of multi-level spatiotemporal simulation of socioeconomic phenomena).

OpenAccess This article is distributed under the terms of the Creative Commons Attribution 4.0 International License (http://creativecommons.org/licenses/by/4.0/), which permits unrestricted use, distribution, and reproduction in any medium, provided you give appropriate credit to the original author(s) and the source, provide a link to the Creative Commons license, and indicate if changes were made.

\section{Appendix}

\section{Properties of function $\theta_{1}(x)$}

Using the unit step function $\theta(d)$ to define a binary value function $\theta_{1}(d)$ :

$$
\theta_{1}(d)=\theta(d-1)=\left\{\begin{array}{cc}
1 & d \geq 1 \\
0 & \text { else }
\end{array}\right.
$$


The following shows prosperities of function $\theta_{1}(d)$ for derivation of equations of spreading on network. In this system, the existence of link $\widetilde{A}_{i j}$ and spreading state of time $(\vec{H}(t))_{i}$ are binary values. All the operations in this study are multiplication and addition without any subtraction. All values in this study should be non-negative integers. We show prosperities of function $\theta_{1}(d)$ for non-negative integers. The symbols $d$ and $e$ are arbitrary non-negative integers, and $\vec{D}, \vec{E}$ are vectors or matrices with arbitrary non-negative integers:

$$
\begin{aligned}
& \left\{\begin{array}{l}
\theta(d \times e-1)=\theta(d-1) \times \theta(e-1) \\
\theta(d+e-1)=\theta(\theta(d-1)+\theta(e-1)-1)
\end{array}\right\} \\
& \quad \rightarrow\left\{\begin{array}{l}
\theta_{1}(d e)=\theta_{1}(d) \theta_{1}(e) \\
\theta_{1}(d+e)=\theta_{1}\left(\theta_{1}(d)+\theta_{1}(e)\right)
\end{array}\right\} \\
& \rightarrow \theta_{1}\left(d_{1} e_{1}+d_{2} e_{2}\right)=\theta_{1}\left(\theta_{1}\left(d_{1} e_{1}\right)+\theta_{1}\left(d_{2} e_{2}\right)\right)=\theta_{1}\left(\theta_{1}\left(d_{1}\right) \theta_{1}\left(e_{1}\right)+\theta_{1}\left(d_{2}\right) \theta_{1}\left(e_{2}\right)\right) .
\end{aligned}
$$

We generalise the scalar function $\theta_{1}$ to be a matrix function

$$
\begin{aligned}
& \left(\theta_{1}(\vec{D})\right)_{i, m}=\theta_{1}\left(\vec{D}_{i, m}\right) \\
& \quad \rightarrow\left\{\begin{array}{l}
\theta_{1}\left(\vec{D}^{T} \vec{E}\right)=\theta_{1}\left(\theta_{1}\left(\vec{D}^{T}\right) \theta_{1}(\vec{E})\right) \\
\theta_{1}(\vec{D}+\vec{E})=\theta_{1}\left(\theta_{1}(\vec{D})+\theta_{1}(\vec{E})\right)
\end{array}\right\} .
\end{aligned}
$$

The values of zero and one are the two fix points of function $\theta_{1}(x): \theta_{1}(0)=0$, $\theta_{1}(1)=1$. For an arbitrary binary matrix or vector $\vec{B}$, which is with element zero or one, $\vec{B}$ will be the same post been acted by $\theta_{1}$ :

$$
\theta_{1}(\vec{B})=\vec{B}
$$

And function value of $\theta_{1}(d)$ is binary, therefore, for arbitrary matrix or vector:

$$
\theta_{1}\left(\theta_{1}(\vec{D})\right)=\theta_{1}(\vec{D})
$$




\section{Detail derivation of Eq. 1}

$$
\begin{aligned}
& \vec{H}(t+1)=\vec{H}(t)+\theta_{1}(\widetilde{A}(t) \vec{H}(t)) \vec{H}(t+1)=\theta_{1}(\vec{H}(t))+\theta_{1}(\widetilde{A}(t) \vec{H}(t)) \\
& \rightarrow \vec{H}(t+1)=\theta_{1}(\vec{H}(t)+\widetilde{A}(t) \vec{H}(t)) \\
&=\theta_{1}((\widetilde{I}+\widetilde{A}(t)) \vec{H}(t)) \\
&=\theta_{1}\left(\theta_{1}(\widetilde{I}+\widetilde{A}(t)) \vec{H}(t)\right) \\
& \rightarrow \vec{H}(t+2)=\theta_{1}\left(\theta_{1}(\widetilde{I}+\widetilde{A}(t-1)) \theta_{1}(\widetilde{I}+\widetilde{A}(t)) \vec{H}(t)\right) \\
& \rightarrow \vec{H}(t)=\theta_{1}\left(\left(\prod_{t^{\prime}=0}^{\curvearrowleft} \theta_{1}\left(\widetilde{I}+\widetilde{A}\left(t^{\prime}\right)\right) \vec{H}(0)\right)\right. \\
& \rightarrow \vec{H}(t)=\theta_{1}\left(\prod_{t^{\prime}=0}^{\curvearrowleft}\left(\widetilde{I}+\widetilde{A}\left(t^{\prime}\right)\right) \vec{H}(0)\right) \\
& \rightarrow \vec{H}(t)=\theta_{1}\left(\prod_{t^{\prime}=0}^{n}\left(\widetilde{I}+\widetilde{A}\left(t^{\prime}\right)\right) \vec{H}(0)\right) .
\end{aligned}
$$

\section{References}

1. Barabsi, A. L. (2015). Network science. Cambridge: Cambridge University Press.

2. Holme, P., \& Saramki, J. (2012). Temporal networks. Physics Reports, 519(3), 97-125. https://doi. org/10.1016/j.physrep.2012.03.001.

3. Mohar, B. (1992). Laplace eigenvalues of graphs: A survey. Discrete Mathematics, 109(1), 171183. https://doi.org/10.1016/0012-365X(92)90288-Q.

4. Seary, A. J., \& Richards, W. D. (2003). Spectral methods for analyzing and visualizing networks: An introduction. In K. C. Ronald Breiger \& P. Pattison (Eds.), Dynamic social network modeling and analysis : Workshop summary and papers (pp. 209-228). Washington: The National Academies.

5. Valdano, E., Poletto, C., Giovannini, A., Palma, D., Savini, L., \& Colizza, V. (2015). Predicting epidemic risk from past temporal contact data. PLOS Computational Biology, 11(3), 1-19. https://doi. org/10.1371/journal.pcbi.1004152.

6. Wang, S. C., \& Ito, N. (2018). Pathogenicdynamic epidemic agent model with an epidemic threshold. Physica A: Statistical Mechanics and Its Applications, 505, 1038-1045. https://doi. org/10.1016/j.physa.2018.04.035.

Publisher's Note Springer Nature remains neutral with regard to jurisdictional claims in published maps and institutional affiliations. 